\title{
Mental disorders among persons with heart disease — results from World Mental Health surveys
}

Johan Ormel, Ph.D. ${ }^{a, *}$, Michael Von Korff, Sc.D. ${ }^{c}$, Huibert Burger, M.D., Ph.D. ${ }^{\mathrm{a}, \mathrm{b}}$, Kate Scott, Ph.D. ${ }^{d}$, Koen Demyttenaere, M.D., Ph.D. ${ }^{\mathrm{e}}$, Yue-qin Huang, M.P.H., M.D., Ph.D. ${ }^{\mathrm{f}}$, José Posada-Villa, M.D. ${ }^{\text {g,h }}$, Jean Pierre Lepine, M.D. ${ }^{i}$, Matthias C. Angermeyer, M.D. ${ }^{j}$, Daphna Levinson, Ph.D. ${ }^{\mathrm{k}}$, Giovanni de Girolamo, M.D. ${ }^{1}$, Norito Kawakami, M.D. ${ }^{\mathrm{m}}$, Elie Karam, M.D. ${ }^{\mathrm{n}}$, María Elena Medina-Mora, Ph.D. ${ }^{\circ}$, Oye Gureje, M.D., Ph.D., FRCPsych ${ }^{\mathrm{p}}$, David Williams, Ph.D., M.P.H. ${ }^{\mathrm{q}}$, Josep Maria Haro, M.D., M.P.H., Ph.D. ${ }^{\mathrm{r}}$, Evelyn J. Bromet, Ph.D. ${ }^{\mathrm{s}}$, Jordi Alonso, M.D., Ph.D. ${ }^{\mathrm{t}}$, Ron Kessler, Ph.D. ${ }^{\mathrm{u}}$

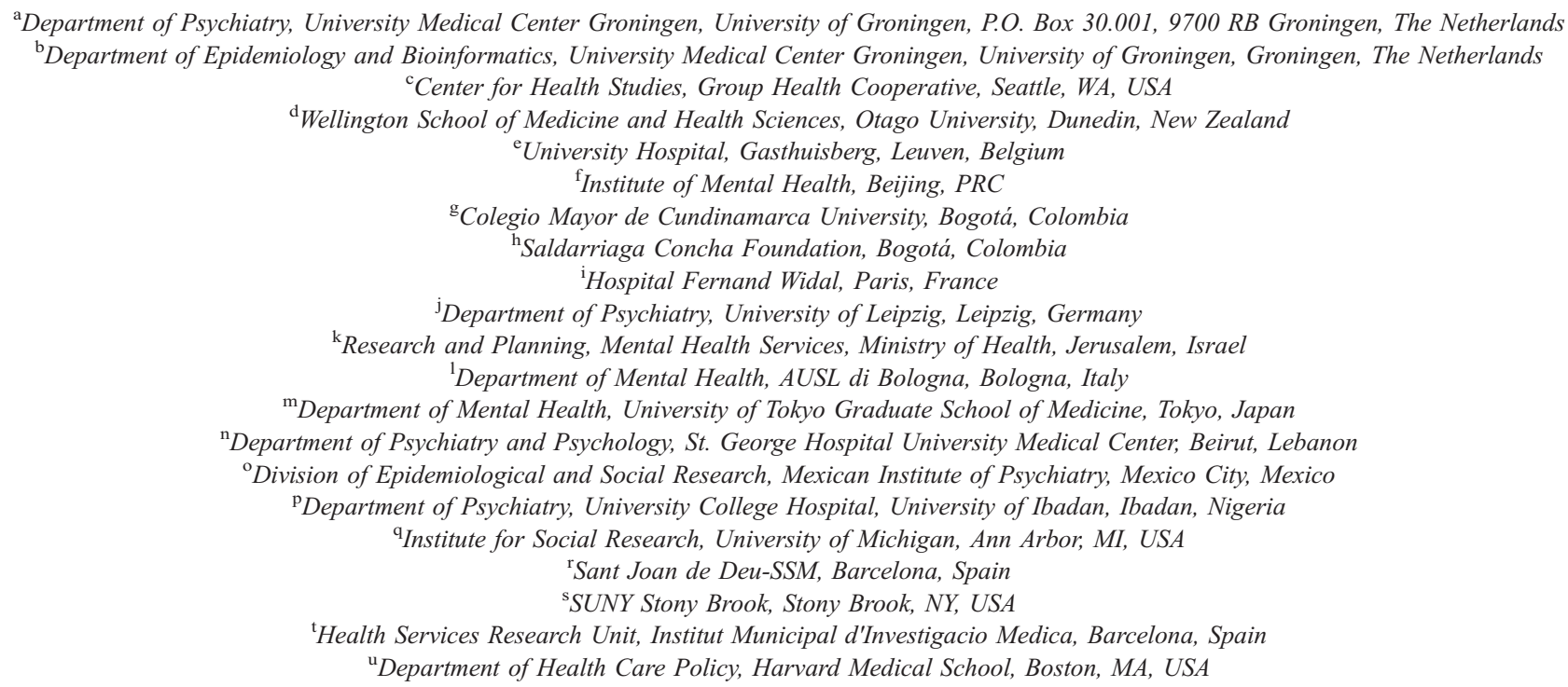

\begin{abstract}
Objectives: While depression and heart disease often co-occur in Western countries, less is known about the association of anxiety and alcohol use disorders with heart disease and about the cross-cultural consistency of this association. Consistency across emotional disorders and cultures would suggest that relatively universal mechanisms underlie the association.

Methods: Surveys with 18 random population samples of household-residing adults in 17 countries in Europe, the Americas, the Middle East, Africa, Asia and the South Pacific were carried out. Medically recognized heart disease was ascertained by self-report. Mental disorders were assessed with the World Mental Health Composite International Diagnostic Interview, a fully structured diagnostic interview.
\end{abstract}

* Corresponding author. Tel.: +31 50361 2078; fax: +31503619722.

E-mail address: j.ormel@med.umcg.nl (J. Ormel). 
Results: Specific mood and anxiety disorders occurred among persons with heart disease at rates higher than those among persons without heart disease. Adjusted for sex and age, the pooled odds ratios (95\% confidence interval) were 2.1 (1.9-2.5) for mood disorders, 2.2 (1.9$2.5)$ for anxiety disorders and $1.4(1.0-1.9)$ for alcohol abuse/dependence among persons with versus those without heart disease. These patterns were similar across countries.

Conclusions: An excess of anxiety disorders and that of mood disorders are found among persons with heart disease. These associations hold true across countries despite substantial between-country differences in culture and mental disorder prevalence rates. These results suggest that similar mechanisms underlie the association and that a broad spectrum of mood-anxiety disorders should be considered in research on the comorbidity of mental disorders and heart disease.

(C) 2007 Elsevier Inc. All rights reserved.

Keywords: Heart disease; Depression; Anxiety; Substance abuse; Cross-national

\section{Introduction}

Depression and ischemic heart disease are leading sources of disease burden worldwide [1-3]. Prior research found that persons with heart disease are more likely to experience depressive illness and that comorbid depression is associated with a twofold or greater increased risk for all-cause mortality, cardiovascular mortality and new cardiovascular events [4-7], but a body of convincing evidence showing that effective treatment of depression improves the prognosis of heart disease is lacking [8-10]. Multiple comorbidity mechanisms have been proposed to explain the association, including health-related behaviors, impairments in autonomic function, elevated levels of proinflammatory cytokines and increased platelet function (e.g., [6,11-14]).

To date, research on heart disease-mental disorder comorbidity had focused on depression and had largely been conducted in Western countries [15-18]. Less is known about the association of heart disease with anxiety or alcohol use disorders or about mental-physical comorbidity in developing countries and non-Western cultures. Using data from World Mental Health (WMH) surveys in 17 countries in Europe, the Americas, Asia, the Middle East and Africa, we provide new information regarding the occurrence of common mental disorders among persons with heart disease and the consistency of the association across diverse Western and non-Western countries. It is important to know such data. If the association between heart disease and mental disorder is not limited to depression and Western countries but consistently observed around the world and for mental disorders other than depression, then the position that relatively universal mechanisms underlie the association would be supported.

Studying mental-physical comorbidity for heart disease via morbidity surveys in a wide range of developing countries necessitated reliance on self-report data. Mental disorders were assessed using a standardized diagnostic instrument. Regarding heart disease, respondents were asked if a medical doctor or any other health professional had ever told them that they had a heart disease. Although the WMH surveys were unable to confirm self-reports of medically diagnosed heart disease by independent means, validity studies had shown that similar measures have acceptable validity when compared with medical records, with $\kappa$ values generally exceeding 0.60 , sensitivities of 0.70 or greater and specificities of approximately 0.95 [19-22]. While it is certainly possible that recall bias due to mental illness affects research on perceived health and symptoms, it is far less so the case in research on medically diagnosed disorders (e.g., $[23,24]$ ). While the limitations of self-report of medically recognized heart disease need to be borne in mind, this article presents new information on the association of heart disease with mood, anxiety and alcohol use disorders in diverse countries worldwide. The objectives of our analysis were to (1) estimate the prevalence of specific mood, anxiety and alcohol use disorders among persons with and those without heart disease and (2) assess whether these associations are consistent across culturally and socioeconomically diverse countries.

\section{Methods}

\subsection{Samples}

Eighteen surveys were carried out in 17 countries in the Americas (Colombia, Mexico and United States), Europe (Belgium, France, Germany, Italy, The Netherlands, Spain and Ukraine), the Middle East/Africa (Israel, Lebanon, Nigeria and South Africa), Asia (Japan and the People's Republic of China, with separate surveys in Beijing and Shanghai) and the South Pacific (New Zealand). All surveys were based on multistage and clustered area probability household samples. All interviews were carried out face to face by trained lay interviewers. The six Western European surveys were carried out jointly.

Internal subsampling was used to reduce respondent burden by dividing the interview into two parts (except for Israel). Part 1 included the core diagnostic assessment of mental disorders, whereas Part 2 included collection of additional information relevant to a wide range of survey aims, including assessment of chronic physical conditions. All respondents completed Part 1. All Part 1 respondents who met the criteria for any mental disorder and a probability sample of other respondents were administered Part 2. The results from Part 2 respondents were weighted by the inverse of their probability of selection for Part 2 of the interview to adjust for differential sampling. Analyses reported in this article were based on the weighted Part 2 sample. Additional weights were used to adjust for differential probabilities of 
selection within households and to match the samples to population sociodemographic distributions. The samples showed substantial cross-national differences in age structure (younger in less developed countries) and educational status (lower in less developed countries) but not in sex distribution.

\subsection{Training and field procedures}

The central WMH staff trained bilingual supervisors in each country. Consistent interviewer training documents and procedures were used across surveys. The World Health Organization (WHO) translation protocol was used to translate instruments and training materials. Standardized descriptions of the goals and procedures of the study, data usage and protection and the rights of respondents were provided in written and verbal forms to all potentially eligible respondents before obtaining their verbal informed consent for participation in the survey. Quality control protocols, described in more detail elsewhere [25], were standardized across countries to check on interviewer accuracy and specify data cleaning as well as coding procedures. The institutional review board of the organization that coordinated the survey in each country approved and monitored compliance with procedures for obtaining informed consent and protecting human subjects.

\subsection{Mental disorder status}

All surveys used the WMH Survey Version 3.0 of the WHO Composite International Diagnostic Interview [26], a fully structured diagnostic interview, to assess disorders and treatments. Disorders considered in this article include anxiety [generalized anxiety disorder (GAD), panic disorder and/or agoraphobia, posttraumatic stress disorder (PTSD) and social phobia], mood (dysthymia and major depressive disorder) and alcohol use (alcohol abuse and dependence) disorders. Disorders were assessed using the definitions and criteria of the Diagnostic and Statistical Manual of Mental Disorders, Fourth Edition [27]. Composite International Diagnostic Interview organic exclusion rules were imposed in making all diagnoses. WHO Composite International Diagnostic Interview field trials and subsequent clinical calibration studies had shown that the 12-month prevalence rates of the disorders considered herein were assessed with acceptable reliability [25].

\subsection{Heart disease status}

In a series of questions about chronic conditions adapted from the U.S. Health Interview Survey [19], respondents were asked about the presence of selected chronic conditions. More specifically, respondents were asked if a medical doctor or any other health professional had ever told them that they had a heart disease. Self-reports of medically diagnosed heart disease have been shown to have acceptable validity, with some underreporting but little overreporting [19-22]. Self-report measures of heart disease have been used previously in research on heart disease and psychopathology [28-31], but the limitations and potential biases of

Table 1

Characteristics of and heart disease prevalence rates for the samples

\begin{tabular}{|c|c|c|c|c|c|c|c|}
\hline \multirow[t]{2}{*}{ Country } & \multirow[t]{2}{*}{ Sample $(n)$} & \multirow{2}{*}{$\begin{array}{l}\text { Mean age } \\
\left(\text { years) }^{\mathrm{a}}\right.\end{array}$} & \multirow{2}{*}{$\begin{array}{l}\text { Aged } 60 \text { years } \\
\text { or older }(\%)\end{array}$} & \multirow[t]{2}{*}{ Women $(\%)$} & \multirow{2}{*}{$\begin{array}{l}\text { Education } \\
\text { ( } \%, \text { secondary } \\
\text { or greater) }\end{array}$} & \multicolumn{2}{|c|}{ Heart disease prevalence rates $^{b}$} \\
\hline & & & & & & $\begin{array}{l}\text { Prevalence } \\
(n)\end{array}$ & $\begin{array}{l}\text { Weighted } \\
\text { average }(\%)\end{array}$ \\
\hline \multicolumn{8}{|l|}{ Americas } \\
\hline Colombia & 2381 & 36.6 & 5.3 & 54.5 & 46.4 & 108 & 3.0 \\
\hline Mexico & 2362 & 35.2 & 5.2 & 52.3 & 31.4 & 78 & 2.3 \\
\hline United States & 5692 & 45.0 & 21.2 & 53.0 & 83.2 & 399 & 7.0 \\
\hline \multicolumn{8}{|c|}{ Asia and South Pacific } \\
\hline Japan & 887 & 51.4 & 34.9 & 53.7 & 70.0 & 70 & 6.1 \\
\hline PRC Beijing & 914 & 39.8 & 15.6 & 47.5 & 61.4 & 129 & 10.1 \\
\hline PRC Shanghai & 714 & 42.9 & 18.7 & 48.1 & 46.8 & 100 & 12.4 \\
\hline New Zealand & 7312 & 44.6 & 20.7 & 52.2 & 60.4 & 504 & 6.7 \\
\hline \multicolumn{8}{|l|}{ Europe } \\
\hline Belgium & 1043 & 46.9 & 27.3 & 51.7 & 69.7 & 105 & 8.9 \\
\hline France & 1436 & 46.3 & 26.5 & 52.2 & NA & 74 & 5.6 \\
\hline Germany & 1323 & 48.2 & 30.6 & 51.7 & 96.4 & 121 & 8.8 \\
\hline Italy & 1779 & 47.7 & 29.2 & 52.0 & 39.5 & 104 & 5.4 \\
\hline The Netherlands & 1094 & 45.0 & 22.7 & 50.9 & 69.7 & 104 & 7.8 \\
\hline Spain & 2121 & 45.5 & 25.5 & 51.4 & 41.7 & 122 & 4.5 \\
\hline Ukraine & 1720 & 46.1 & 27.3 & 55.1 & 79.5 & 582 & 25.0 \\
\hline \multicolumn{8}{|c|}{ Middle East and Africa } \\
\hline Lebanon & 602 & 40.3 & 15.3 & 48.1 & 40.5 & 31 & 3.3 \\
\hline Nigeria & 2143 & 35.8 & 9.7 & 51.0 & 35.6 & 36 & 1.7 \\
\hline Israel & 4859 & 44.4 & 20.3 & 51.9 & 78.3 & 511 & 9.4 \\
\hline South Africa & 4315 & 37.1 & 8.8 & 53.6 & 38.9 & 297 & 6.1 \\
\hline
\end{tabular}

NA, not available.

${ }^{a}$ Minimum age is 18 years, except for Colombia, Mexico (age range, 18-65 years), Japan (minimum age, 20 years) and Israel (minimum age, 21 years).

b Lifetime prevalence reported. 
Table 2

Prevalence rates of mood disorders among persons with versus those without heart disease

\begin{tabular}{|c|c|c|c|c|c|c|}
\hline \multirow[t]{2}{*}{ Country } & \multicolumn{3}{|c|}{ Major depression } & \multicolumn{3}{|l|}{ Dysthymia } \\
\hline & $\begin{array}{l}\text { Without heart } \\
\text { disease }(\%)\end{array}$ & $\begin{array}{l}\text { With heart } \\
\text { disease }(\%)\end{array}$ & OR (CI) & $\begin{array}{l}\text { Without heart } \\
\text { disease }(\%)\end{array}$ & $\begin{array}{l}\text { With heart } \\
\text { disease }(\%)\end{array}$ & OR (CI) \\
\hline Colombia & 5.9 & 13.9 & $2.6(1.5-4.8) *$ & 0.9 & 5.0 & $5.6(1.8-17.4) *$ \\
\hline Mexico & 4.0 & 8.7 & $2.1(1.0-4.5)$ & 0.9 & 0.9 & $0.8(0.2-3.9)$ \\
\hline United States & 8.2 & 9.2 & $2.1(1.4-3.2) *$ & 2.2 & 3.7 & $2.6(1.5-4.8) *$ \\
\hline Japan & 2.1 & 4.6 & $3.2(1.1-8.9) *$ & 0.6 & 2.7 & $4.6(1.0-20.7) *$ \\
\hline PRC Beijing & 2.3 & 3.1 & $2.0(1.0-4.0)$ & 0.3 & 0.9 & $2.0(0.4-9.4)$ \\
\hline PRC Shanghai & 0.9 & 6.9 & $7.4(1.9-29.4) *$ & 0.4 & 0.2 & $0.3(0.0-4.8)$ \\
\hline New Zealand & 6.8 & 4.2 & $1.4(0.9-2.3)$ & 1.8 & 1.9 & $1.8(1.0-3.3)$ \\
\hline Belgium & 5.6 & 5.6 & $1.9(0.7-5.6)$ & 1.1 & 3.0 & $2.3(0.7-7.4)$ \\
\hline France & 6.1 & 6.4 & $2.1(0.7-5.8)$ & 1.6 & 1.4 & $0.9(0.2-3.5)$ \\
\hline Germany & 3.0 & 3.3 & $2.0(0.7-6.1)$ & 0.7 & 3.1 & $4.2(1.1-15.9) *$ \\
\hline Italy & 3.0 & 5.7 & $2.0(1.0-4.3)$ & 0.8 & 4.8 & $4.3(2.2-8.3) *$ \\
\hline The Netherlands & 5.1 & 7.4 & $2.8(1.0-7.8) *$ & 1.7 & 2.9 & $3.8(0.4-37.8)$ \\
\hline Spain & 3.9 & 7.4 & $2.3(1.1-4.8) *$ & 1.3 & 4.1 & $3.3(1.3-8.5) *$ \\
\hline Ukraine & 6.4 & 18.6 & $2.5(1.8-3.5) *$ & 2.5 & 9.0 & $2.3(1.6-3.3) *$ \\
\hline Lebanon & 1.8 & 1.3 & $1.2(0.2-6.2)$ & 0.7 & 0.0 & $\mathrm{NE}$ \\
\hline Nigeria & 1.1 & 2.4 & $2.3(0.8-6.5)$ & 0.2 & 0.4 & $1.9(0.2-21.6)$ \\
\hline Israel & 5.9 & 8.0 & $1.6(1.0-2.4) *$ & 1.1 & 2.1 & $1.7(0.8-3.5)$ \\
\hline South Africa & 4.5 & 10.3 & $2.2(1.4-3.6) *$ & 0.1 & 0.0 & $\mathrm{NE}$ \\
\hline Pooled OR & - & - & $2.1(1.8-2.4) *$ & - & - & $2.4(2.0-3.0) *$ \\
\hline
\end{tabular}

NE, nonestimable.

$* P<.05$.

self-report of medically recognized heart disease need to be considered in interpreting the results of the WMH surveys.

\subsection{Data analysis}

This article reports on prevalence rates for specific mental disorders among persons with and those without heart disease. For each survey, all odds ratios (ORs) quantifying the association of each mental disorder with heart disease were estimated while adjusting for age and sex. Ninety-five percent confidence intervals (CIs) for the prevalence rates and for the ORs were estimated using the Taylor series method [32] with SUDAAN software [33] to adjust for clustering and weighting. Pooled estimates of mental disorder prevalence rates are not reported due to the large variation in mental disorder prevalence rates across surveys.

Using meta-analytical methods, we calculated pooled estimates of the ORs from the 18 surveys, describing the overall association of each mental disorder with heart disease. The pooled estimate of the OR was weighted by the inverse of the variance of the estimate for each survey. We report on the CIs of the pooled OR estimates [34]. For each association of a specific mental disorder with heart disease, we assessed whether the heterogeneity of the OR estimates across surveys was greater than that expected by chance [34]. Since these tests were nonsignificant except for agoraphobia/panic disorder, we report on pooled estimates of the ORs and CIs for those pooled estimates.

The adjusted ORs and the pooled estimates as well as their CIs are displayed for each survey using a funnel graph [35]. The funnel in these graphs shows the $95 \% \mathrm{CI}$ band for a survey estimate that would include the pooled estimate of the
$\mathrm{OR}$ at varying levels of precision. Precision is defined as the reciprocal of the standard error of the OR estimate. On these graphs, the less precise estimates are on the left (where the funnel is wider) and the more precise estimates are on the right (where the funnel is narrower). These graphs provide a visual summary of the association of any mood disorder and that of any anxiety disorder with heart disease across the participating surveys.

The prevalence of heart disease is so low among younger people that the statistical adjustment for age may not be entirely without risk as it might obscure differences in the strength of the associations of interest between younger and older respondents. Therefore, we reran the analyses described above for respondents aged 50 years or older.

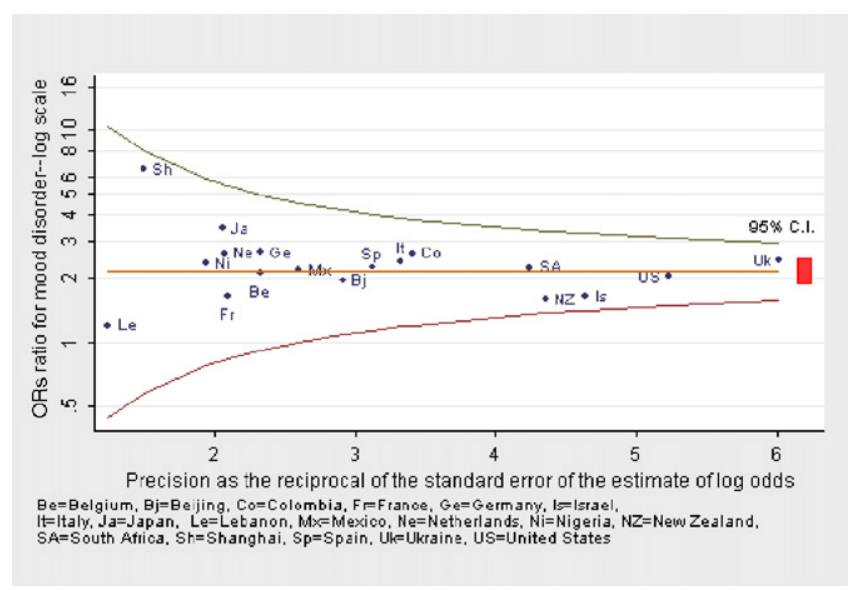

Fig. 1. ORs (age and sex adjusted) for mood disorder among persons with versus those without heart disease. 


\section{Results}

\subsection{Sample characteristics}

Survey sample sizes ranged from 2372 (The Netherlands) to 12,992 (New Zealand), with a total of 85,052 participating adults in Part 1 and that of 43,249 in Part 2. Response rates ranged from $45.9 \%$ (France) to $87.7 \%$ (Colombia), with a sample size weighted average of $70.5 \%$ (Table 1 ).

Self-reported heart disease was common in all of the participating countries. Excluding Ukraine, where the prevalence of self-reported heart disease was exceptionally high (25\%), prevalence rates ranged from $1.7 \%$ in Nigeria to $12.4 \%$ in Shanghai, suggesting substantial across-country variation (Table 1). European, Asian and North American countries had a higher prevalence of heart disease as compared with South American, Arabic Middle East and African countries. Prevalence was higher among older persons, among those with less than 12 years of education and generally among men (data available upon request). Exceptions to this pattern included Ukraine (31.0\% prevalence for females vs. $17.5 \%$ for males) and Beijing (12.7\% prevalence for females vs. $7.7 \%$ for males).

Table 3

Prevalence rates of anxiety disorders among persons with versus those without heart disease

\begin{tabular}{|c|c|c|c|c|c|c|}
\hline \multirow[t]{2}{*}{ Country } & \multicolumn{3}{|c|}{ Generalized anxiety } & \multicolumn{3}{|c|}{ Agoraphobia or panic disorder } \\
\hline & $\begin{array}{l}\text { Without heart } \\
\text { disease }(\%)\end{array}$ & $\begin{array}{l}\text { With heart } \\
\text { disease }(\%)\end{array}$ & OR (CI) & $\begin{array}{l}\text { Without heart } \\
\text { disease }(\%)\end{array}$ & $\begin{array}{l}\text { With heart } \\
\text { disease }(\%)\end{array}$ & OR (CI) \\
\hline Colombia & 1.0 & 1.5 & $1.6(0.4-6.3)$ & 2.1 & 4.9 & $2.5(1.0-6.3)$ \\
\hline Mexico & 0.5 & 1.0 & $1.7(0.2-11.6)$ & 1.3 & 0.6 & $0.4(0.1-1.9)$ \\
\hline United States & 4.0 & 4.9 & $1.9(1.2-3.0)^{*}$ & 3.6 & 4.0 & $2.0(1.3-3.0)^{*}$ \\
\hline Japan & 1.4 & 4.2 & $3.1(0.9-10.9)$ & 0.5 & 2.8 & $15.9(4.4-58.0) *$ \\
\hline PRC Beijing & 1.0 & 2.4 & $2.0(0.9-4.5)$ & 0.4 & 0.3 & $0.7(0.0-12.0)$ \\
\hline PRC Shanghai & 0.2 & 4.6 & $12.3(2.4-62.4) *$ & 0.0 & 1.0 & $\mathrm{NE}$ \\
\hline New Zealand & 3.1 & 2.6 & $1.6(0.9-2.6)$ & 2.2 & 2.2 & $2.4(1.2-4.6) *$ \\
\hline Belgium & 1.1 & 0.5 & $0.7(0.2-2.7)$ & 1.6 & 0.0 & - \\
\hline France & 1.9 & 5.3 & $5.7(0.8-40.7)$ & 1.2 & 3.8 & $6.4(1.7-24.7) *$ \\
\hline Germany & 0.4 & 1.3 & $10.5(1.5-74.0) *$ & 1.1 & 0.7 & $1.5(0.3-7.5)$ \\
\hline Italy & 0.5 & 0.8 & $2.0(0.3-16.5)$ & 1.0 & 1.8 & $2.2(0.4-11.0)$ \\
\hline The Netherlands & 1.1 & 0.5 & $0.5(0.0-6.0)$ & 1.6 & 2.5 & $3.0(0.9-10.0)$ \\
\hline Spain & 0.9 & 1.8 & $2.1(0.6-7.5)$ & 0.8 & 1.7 & $2.6(0.7-8.9)$ \\
\hline Ukraine & 1.3 & 5.4 & $3.3(2.1-5.4) *$ & 0.9 & 4.7 & $4.9(2.9-8.2) *$ \\
\hline Lebanon & 0.2 & 0.3 & $1.7(0.2-15.3)$ & 0.2 & 0.0 & $\mathrm{NE}$ \\
\hline Nigeria & 0.0 & 0.0 & $\mathrm{NE}$ & 0.2 & 6.0 & $32.6(4.5-234.4) *$ \\
\hline Israel & 2.6 & 2.8 & $1.1(0.6-2.1)$ & 0.9 & 1.5 & $1.5(0.7-3.4)$ \\
\hline South Africa & 1.7 & 5.1 & $2.3(1.1-4.9) *$ & 5.1 & 13.0 & $2.7(1.7-4.1) *$ \\
\hline Pooled OR & - & - & $2.1(1.7-2.5)^{*}$ & - & - & $2.7(2.2-3.3) *$ \\
\hline \multirow[t]{2}{*}{ Country } & \multicolumn{3}{|l|}{ Social phobia } & \multicolumn{3}{|l|}{ PTSD } \\
\hline & $\begin{array}{l}\text { Without heart } \\
\text { disease }(\%)\end{array}$ & $\begin{array}{l}\text { With heart } \\
\text { disease (\%) }\end{array}$ & OR (CI) & $\begin{array}{l}\text { Without heart } \\
\text { disease }(\%)\end{array}$ & $\begin{array}{l}\text { With heart } \\
\text { disease (\%) }\end{array}$ & OR (CI) \\
\hline Colombia & 2.8 & 4.8 & $2.1(0.8-5.1)$ & 0.6 & 0.5 & $0.9(0.1-5.7)$ \\
\hline Mexico & 1.9 & 5.0 & $2.9(1.1-7.7)^{*}$ & 0.6 & 1.1 & $2.5(0.6-10.7)$ \\
\hline United States & 6.9 & 6.0 & $1.6(1.0-2.6)$ & 3.5 & 4.8 & $2.7(1.7-4.3)^{*}$ \\
\hline Japan & 0.5 & 2.0 & $9.3(1.7-51.2) *$ & 0.4 & 0.0 & $\mathrm{NE}$ \\
\hline PRC Beijing & 0.3 & 0.7 & $5.3(1.2-24.1)^{*}$ & 0.2 & 0.8 & $3.3(0.5-23.3)$ \\
\hline PRC Shanghai & 0.0 & 0.0 & $\mathrm{NE}$ & 0.1 & 0.4 & $6.8(0.4-124.2)$ \\
\hline New Zealand & 5.2 & 2.4 & $0.9(0.5-1.6)$ & 3.0 & 3.0 & $1.5(0.9-2.5)$ \\
\hline Belgium & 1.2 & 0.4 & $1.6(0.1-18.9)$ & 0.7 & 0.8 & $2.7(0.8-9.0)$ \\
\hline France & 2.7 & 1.6 & $1.0(0.1-7.7)$ & 2.3 & 2.4 & $2.2(0.4-11.7)$ \\
\hline Germany & 1.9 & 0.6 & $0.8(0.2-3.6)$ & 0.7 & 0.7 & $3.2(0.7-15.2)$ \\
\hline Italy & 1.0 & 2.8 & $4.2(0.9-19.1)$ & 0.7 & 1.6 & $1.9(0.4-8.9)$ \\
\hline The Netherlands & 1.2 & 2.4 & $4.2(1.2-14.7)^{*}$ & 2.3 & 4.8 & $2.5(0.4-14.1)$ \\
\hline Spain & 0.7 & 0.9 & $4.0(0.3-52.8)$ & 0.5 & 0.7 & $1.5(0.3-6.3)$ \\
\hline Ukraine & 2.0 & 2.2 & $1.8(0.9-4.0)$ & 1.6 & 6.3 & $3.3(1.9-5.6)^{*}$ \\
\hline Lebanon & 0.6 & 0.0 & $\mathrm{NE}$ & 1.7 & 0.9 & $1.1(0.1-10.6)$ \\
\hline Nigeria & 0.3 & 0.0 & $\mathrm{NE}$ & 0.0 & 0.0 & $\mathrm{NE}$ \\
\hline Israel & - & - & $\mathrm{NE}$ & 0.5 & 0.8 & $1.5(0.3-7.0)$ \\
\hline South Africa & 1.7 & 5.0 & $3.4(1.6-7.0) *$ & 0.6 & 0.9 & $1.5(0.3-7.4)$ \\
\hline Pooled OR & - & - & $1.9(1.5-2.5)^{*}$ & - & - & $2.3(1.8-2.9) *$ \\
\hline
\end{tabular}

NE, nonestimable. $* P<.05$. 


\subsection{Mood disorders and heart disease}

Major depression was typically common among persons with heart disease (Table 2) and generally fell in the range of $3-9 \%$. The prevalence rates of dysthymia were typically much lower, generally falling in the vicinity of $1-3 \%$. Comparisons of the prevalence rates of major depression and dysthymia among persons with versus those without heart disease showed small absolute differences in many countries, with a few countries showing slightly lower prevalence rates $(<1 \%)$ among persons with heart disease (e.g., Lebanon), but these unadjusted comparisons did not take age and sex differences of persons with versus those without heart disease into account.

As shown in Table 2, age- and sex-adjusted ORs measuring the association of major depression with heart disease were significantly greater than 1.0 (indicating a positive association greater than that expected by chance) for 9 of the 18 surveys for which ORs were calculated. The remaining ORs were all larger than 1.0. The ORs also indicated a significant association between heart disease and dysthymia for 7 of the 16 surveys for which ORs were estimated. We assessed whether the variability in the OR estimates across surveys was greater than that expected by chance [34]. The resulting test of heterogeneity was nonsignificant for major depression $(P=.83)$ and dysthymia $(P=.38)$, thus making it appropriate to report on pooled estimates. The pooled estimate of the OR for major depression was 2.1, while the pooled estimate of the OR for dysthymia was 2.4 . In the subgroup of older respondents ( $\geq 50$ years), these ORs were 1.9 and 2.3 , respectively.

Fig. 1 shows a funnel graph of the age- and sex-adjusted ORs for mood disorders (major depression and/or dysthymia present) for all 18 surveys. In this graph, the OR is plotted on a log scale as a function of the precision of the estimate of the OR. The funnel lines show whether the 95\% CI of each survey's estimate of the OR includes the pooled estimate, given the precision of the survey's estimate. Most of the OR

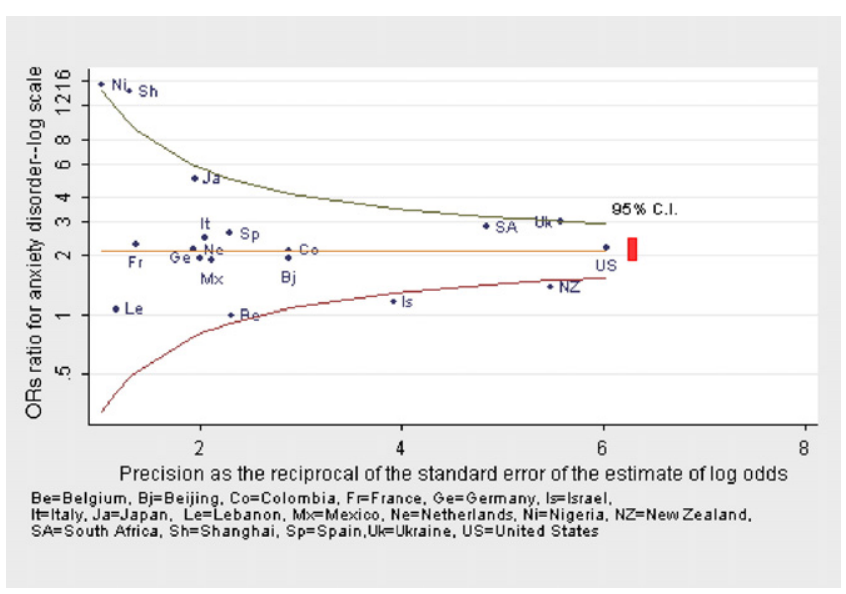

Fig. 2. ORs (age and sex adjusted) for anxiety disorder among persons with versus those without heart disease.

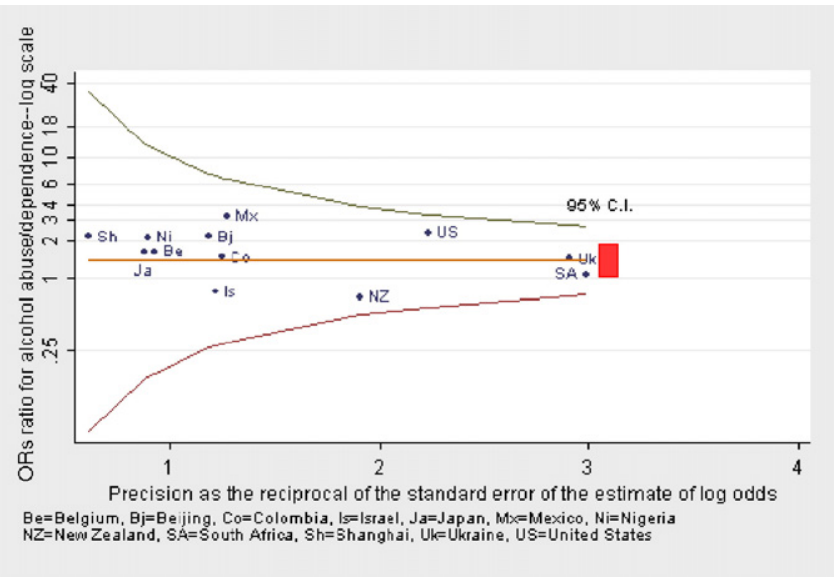

Fig. 3. ORs (age and sex adjusted) for alcohol abuse/dependence among persons with versus those without heart disease.

estimates clustered in proximity to the pooled estimate of 2.1. The $95 \%$ CIs of all of the survey estimates included the pooled estimate, with the more discrepant estimates tending to be those with lower precision.

\subsection{Anxiety disorders and heart disease}

Across the surveys, the specific anxiety disorders (GAD, panic disorder/agoraphobia, social phobia and PTSD) were generally less prevalent as compared with major depression. Among persons with heart disease, the prevalence rates of GAD ranged from approximately $0.3 \%$ in Lebanon to approximately $5 \%$ in Ukraine, the United States and France (Table 3). The prevalence of agoraphobic/panic disorder among persons with heart disease typically fell in the range of $1-5 \%$ (Table 3). Social phobia was rarely found among persons with heart disease in Beijing, Shanghai, Belgium, Germany, Lebanon and Nigeria. The prevalence rates of social phobia ranged from 1\% (Spain) to 6\% (United States) among persons with heart disease in the remaining surveys (Table 3). PTSD was relatively uncommon among persons with heart disease in many of the participating surveys but had a prevalence of $2 \%$ or higher in the United States, France, Italy, The Netherlands and Ukraine (Table 3).

Because the specific anxiety disorders were less common, ORs were not estimated for all of the participating surveys due to a null cell. Given the relatively small sample sizes, it is not surprising that the OR estimates were often not significantly different from 1.0 , even when the OR estimates were consistent with the pooled estimates. The ORs for anxiety disorders tended to be greater than 1.0, although the point estimates showed greater variability than they did for mood disorders, possibly due to the lower sample size of cases. The heterogeneity tests for the ORs were nonsignificant for $\operatorname{GAD}(P=.12)$, social phobia $(P=.08)$ and PTSD $(P=.90)$ but significant for agoraphobia/panic disorder $(P=.003)$, indicating that the pooled estimate for this disorder masks significant heterogeneity across surveys. Across the four anxiety disorders, the pooled OR estimates 
were all significantly greater than 1.0 , falling in the range of 1.9-2.7. In the subgroup of older respondents $(\geq 50$ years old), the ORs were also statistically significant and ranged from 1.5 to 2.6 .

The association of any anxiety disorder with heart disease (Fig. 2) showed a pattern similar to that observed for any mood disorder (Fig. 1). The pooled estimate of the OR was 2.2 , with a $95 \%$ CI of 1.9 to 2.5 . Of the 18 survey-specific estimates, 14 had 95\% CIs that included the pooled estimate. Of the 4 estimates whose $95 \%$ CIs did not include the pooled estimate, 2 had low precision (Nigeria and Shanghai) but 2 others had high precision (Israel and New Zealand). Overall, these results indicate that the strength of the association of anxiety disorders, as a class, with heart disease is comparable with that observed for mood disorders - an odds of about $2: 1$ of anxiety disorder for persons with versus those without heart disease.

\subsection{Alcohol use disorders and heart disease}

In 11 of the 18 surveys, as shown in Fig. 3 and Table 4, $1 \%$ or less of those with heart disease met the criteria for alcohol abuse or dependence. In the remaining surveys, the prevalence rates of alcohol abuse or dependence ranged from 1.4\% (Japan) to about 4.5\% (Mexico and Ukraine). ORs for the association of alcohol abuse or dependence with heart disease were estimated for 12 surveys. These ORs were nearly all larger than 1.0 (except for New Zealand and Israel), albeit not significantly. Since the prevalence of alcohol use disorders decreases markedly with age but the prevalence of heart disease increases with age, adjustment for age and sex is important in assessing the associations. The OR estimates were not found to be heterogeneous across

Table 4

Prevalence rates of substance use disorders among persons with versus those without heart disease

\begin{tabular}{llll}
\hline Country & \multicolumn{3}{l}{ Alcohol abuse/dependence } \\
\cline { 2 - 3 } & $\begin{array}{l}\text { Without heart } \\
\text { disease }(\%)\end{array}$ & $\begin{array}{l}\text { With heart } \\
\text { disease }(\%)\end{array}$ & OR (CI) \\
\hline Colombia & 2.5 & 3.1 & $1.5(0.3-7.3)$ \\
Mexico & 2.2 & 4.2 & $3.2(0.7-15.7)$ \\
United States & 3.2 & 2.2 & $2.4(1.0-5.8)$ \\
Japan & 1.1 & 1.4 & $1.6(0.2-16.4)$ \\
PRC Beijing & 2.6 & 1.6 & $2.2(0.4-12.5)$ \\
PRC Shanghai & 0.4 & 0.6 & $2.2(0.1-64.7)$ \\
New Zealand & 3.0 & 0.4 & $0.7(0.2-1.9)$ \\
Belgium & 1.3 & 1.0 & $1.6(0.2-15.4)$ \\
France & 0.8 & 0.0 & $\mathrm{NE}$ \\
Germany & 1.3 & 0.0 & $\mathrm{NE}$ \\
Italy & 0.1 & 0.0 & $\mathrm{NE}$ \\
The Netherlands & 1.8 & 0.0 & $\mathrm{NE}$ \\
Spain & 0.3 & 0.0 & $\mathrm{NE}$ \\
Ukraine & 6.7 & 4.6 & $1.4(0.7-2.9)$ \\
Lebanon & 1.1 & 0.0 & $\mathrm{NE}$ \\
Nigeria & 0.7 & 0.9 & $2.2(0.2-20.1)$ \\
Israel & 1.2 & 0.4 & $0.8(0.2-3.8)$ \\
South Africa & 5.0 & 3.6 & $1.1(0.5-2.1)$ \\
Pooled OR & - & - & $1.0-1.9)$ \\
\hline
\end{tabular}

surveys $(P=.86)$. The pooled estimate of the OR for the association of alcohol abuse or dependence and heart disease was 1.4 , with a CI of 1.0 to 1.9 . This marginally significant association was not significant in the subgroup of older respondents $(\mathrm{OR}=0.8)$. The $95 \% \mathrm{CIs}$ of the survey-specific estimates of the adjusted ORs for the association of heart disease and alcohol abuse/dependence all included the pooled estimates of the ORs. These results suggest that alcohol abuse may occur with greater frequency among persons with heart disease, although the results do not indicate a strong association.

\section{Discussion}

This report provides the first assessment of the frequency and association of common mental disorders with selfreported medically diagnosed heart disease in representative population samples from diverse countries worldwide. Two key findings emerged from this study: First, the well-known association of depression with heart disease, which was replicated here, was actually not stronger than the association of anxiety disorders with heart disease. This held true for the total population and for respondents aged 50 years or older. The associations persist when adjusted for comorbidity of depressive and anxiety disorders (data not presented but available upon request). Second, since the participating surveys included countries that differed markedly in culture, language, level of socioeconomic development and prevalence of heart disease and mental disorders, the findings hold true across diverse populations despite concerns about differences in the validity of self-report of medically recognized heart disease that may exist between developed and developing countries.

Although the cross-sectional WMH surveys are causally noninformative, the key findings have implications for current thinking about the depression-heart disease association.

Various biobehavioral mechanisms have been proposed to explain this association, including behavioral risk behaviors (e.g., smoking, heavy alcohol use and physical inactivity), poor treatment compliance, elevated levels of proinflammatory cytokines, platelet activation, disturbances in the autonomic nervous system (reduced heart rate variability), hypothalamic-pituitary-adrenal axis dysfunction and the stressfulness of heart disease-related events such as acute myocardial infarction [4-6]. Our results suggest that the implications of anxiety disorders for causal processes may need to be given equal weight with those involving depressive disorders. For instance, one proposed mechanism is poor treatment compliance among depressed heart disease patients, but it has been noted that anxiety disorders are associated with good treatment compliance [36]. Other mechanisms may need to be considered in light of the comorbidity of heart disease with anxiety disorders as well as mood disorders. 
While self-reports of medically diagnosed heart disease have generally good validity, with $\kappa$ values exceeding 0.60 , considerable underreporting and some overreporting of medically recorded heart disease have typically been found [19-22]. Since the WMH surveys asked specifically for medically diagnosed heart disease, it is less likely that respondents reported atypical chest pain [37] and syndrome $X$ [38], although such an error cannot be excluded in a questionnaire survey. Whether any misclassification is nondifferential or produced significant bias is difficult to determine as conclusive evidence either way is lacking. One prior study found that negative affectivity (a trait associated with psychological distress) is associated with self-report of physical symptoms but not with self-report of medically confirmed chronic disease [23]. The results for the depression-heart disease association reported here agree well with those from prior studies in developed countries that used objectively confirmed heart disease measures. This suggests that it is reasonable to place some confidence in the validity of the association of anxiety disorders with heart disease in developed countries. This confidence may extend to less developed countries since the patterns of association of depression and anxiety disorders did not differ notably between the two types of countries. All these do not negate that the self-report of medically recognized heart disease may differ in validity in developed versus less developed countries. Neither can we rule out the possibility that those with mental disorder were more likely to seek care for heart disease symptoms and be labeled as having a heart disease. This treatment seeking bias (Berkson's bias) may have inflated associations. Given the overall pattern of results, we think it is possible that misclassification is largely nondifferential and that underestimation of the association between heart disease and mental disorder is slightly more likely than serious bias. The limitations of these survey results invite future research with more rigorous assessment of heart disease status in developing countries than was possible in this series of studies.

Another limitation of the WMH surveys is the lack of more specific assessments of heart disease in terms of the nature of the heart disease (e.g., rheumatic heart disease and alcoholic cardiomyopathy) and the course of the disease (e.g., post-myocardial infarction). More specific assessments will also improve comparability with available studies that focused on specific heart diseases and on specific phases in their course.

The prevalence of heart disease in the WMH surveys conformed to expected epidemiological patterns (i.e., increasing prevalence with age, lower prevalence in relatively poor countries and higher prevalence in males in general). Also, the exceedingly high prevalence of heart disease observed for Ukraine is in line with findings of previous studies that many Eastern European countries show cardiovascular disease rates that are among the highest in the world [39]. However, we have no ready explanation for the higher prevalence of heart disease in females as compared with males in Ukraine, but cultural or linguistic factors might be involved [40]. The 12month prevalence estimates of major depression among persons with heart disease in Western countries were generally lower than had been reported in prior research [6]. This probably has two causes: First, the WMH study used general population samples rather than persons identified in health care settings, in which the prevalence of depression is higher. Second, the WMH study assessed mental disorders according to a standardized diagnostic instrument rather than the percentage exceeding a predefined threshold on a selfreport depression symptom scale.

\section{Conclusions}

The WMH surveys found that not only mood but also anxiety disorders - as well as alcohol abuse disorders to a lesser extent - occurred among persons with heart disease at higher rates as compared with persons of comparable age and sex without heart disease. This association was observed across diverse countries differing in culture, language, level of socioeconomic development and prevalence of mental disorders. While this research does not shed light on whether psychological disorders are causes or consequences of heart disease, it does suggest that efforts to understand causal relationships between heart disease and psychological illness should consider culture-independent mechanisms that hold true for mood and anxiety disorders. Since mood and anxiety disorders are associated with many different chronic physical conditions (e.g., [6]), the depression-heart disease link also needs to be examined in the broader context of chronic medical disease and psychological illness.

\section{Acknowledgment}

WMH The current report was prepared in conjunction with the WHO WMH Survey Initiative. We thank the WMH staff for their assistance with instrumentation, fieldwork and data analysis. These activities were supported by the United States National Institute of Mental Health (R01MH070884), the John D. and Catherine T. MacArthur Foundation, the Pfizer Foundation, the U.S. Public Health Service (R13-MH066849, R01-MH069864 and R01 DA016558), the Fogarty International Center (FIRCA R01TW006481), the Pan American Health Organization, Eli Lilly and Company, Ortho-McNeil Pharmaceutical, GlaxoSmithKline and Bristol-Myers Squibb. A complete list of WMH publications can be found at http://www.hcp.med.harvard.edu/wmh/.

China The Chinese WMH Survey Initiative was supported by the Pfizer Foundation.

Colombia The Colombian National Study of Mental Health was supported by the Ministry of Social Protection, with supplemental support from the Saldarriaga Concha Foundation.

ESEMeD The ESEMeD project was funded by the European Commission

Europe (Contracts QLG5-1999-01042 and SANCO 2004123), the Piedmont Region (Italy), Fondo de Investigación Sanitaria, Instituto de Salud Carlos III-Spain (FIS 00/0028), Ministerio de Ciencia y Tecnología-Spain (SAF 2000-158-CE), Departament de Salut, Generalitat de Catalunya-Spain and other local agencies as well as by an unrestricted educational 
grant from GlaxoSmithKline.

The Israel National Health Survey was funded by the Ministry of Health with support from the Israel National Institute for Health Policy and Health Services Research and the National Insurance Institute of Israel.

Japan The WMH Japan Survey was supported by a Grant for Research on Psychiatric and Neurological Diseases and Mental Health (H13-SHOGAI-023, H14-TOKUBETSU-026, H16KOKORO-013) from the Japan Ministry of Health, Labor and Welfare.

Lebanon The Lebanese National Mental Health Survey was supported by the Lebanese Ministry of Public Health, the WHO-Lebanon, anonymous private donations to the Institute for Development of Research and Applied Care-Lebanon and unrestricted grants from Janssen Cilag, Eli Lilly and Company, GlaxoSmithKline, Roche and Novartis.

Mexico The Mexican National Comorbidity Survey was supported by the National Institute of Psychiatry Ramon de la Fuente (INPRFMDIES 4280) and the National Council on Science and Technology (CONACyT-G30544-H), with supplemental support from the Pan American Health Organization.

New Te Rau Hinengaro: The New Zealand Mental Health Survey

Zealand was supported by the New Zealand Ministry of Health, Alcohol Advisory Council and the Health Research Council.

Nigeria The Nigerian Survey of Mental Health and Well-Being was supported by the WHO-Geneva, the WHO-Nigeria and the Federal Ministry of Health (Abuja, Nigeria).

South The South Africa Stress and Health Study was supported by the Africa U.S. National Institute of Mental Health (R01-MH059575) and National Institute of Drug Abuse, with supplemental funding from the South African Department of Health and the University of Michigan.

Ukraine The Ukraine Comorbid Mental Disorders During Periods of Social Disruption Study was funded by the U.S. National Institute of Mental Health (RO1-MH61905).

United The U.S. National Comorbidity Survey-Replication was States supported by the National Institute of Mental Health (U01MH60220), with supplemental support from the National Institute of Drug Abuse, the Substance Abuse and Mental Health Services Administration, the Robert Wood Johnson Foundation (Grant 044708) and the John W. Alden Trust.

\section{References}

[1] Insel TR, Collins FS. Psychiatry in the genomics era. Am J Psychiatry 2003;160:616-20.

[2] Ormel J, Von Korff M, Ustun TB, Pini S, Korten A, Oldehinkel T. Common mental disorders and disability across cultures. Results from the WHO Collaborative Study on Psychological Problems in General Health Care. JAMA 1994;272:1741-8.

[3] Murray CJ, Lopez AD. Regional patterns of disability-free life expectancy and disability-adjusted life expectancy: Global Burden of Disease Study. Lancet 1997;349:1347-52.

[4] Rudisch B, Nemeroff CB. Epidemiology of comorbid coronary artery disease and depression. Biol Psychiatry 2003;54:227-40.

[5] Lett HS, Blumenthal JA, Babyak MA, et al. Depression as a risk factor for coronary artery disease: evidence, mechanisms, and treatment. Psychosom Med 2004;66:305-15.

[6] Evans DL, Charney DS, Lewis L, et al. Mood disorders in the medically ill: scientific review and recommendations. Biol Psychiatry 2005;58:175-89.

[7] van Melle JP, de Jonge P, Spijkermanet TA, et al. Prognostic association of depression following myocardial infarction with mortality and cardiovascular events: a meta-analysis. Psychosom Med 2004;66:814-22.
[8] Berkman LF, Blumenthal J, Burg M, et al. Effects of treating depression and low perceived social support on clinical events after myocardial infarction: the Enhancing Recovery in Coronary Heart Disease Patients (ENRICHD) Randomized Trial. JAMA 2003;289:3106-16.

[9] Glassman AH, O'Connor CM, Califf RM, et al. Sertraline treatment of major depression in patients with acute MI or unstable angina. JAMA 2002;288:701-9.

[10] Strik JJMH, Honig A, Lousberg R, et al. Efficacy and safety of fluoxetine in the treatment of patients with major depression after first myocardial infarction: findings from a double-blind placebo-controlled trial. Psychosom Med 2000;62:783-9.

[11] Carney RM, Freedland KE, Jaffe AS. Depression as a risk factor for coronary heart disease mortality. Arch Gen Psychiatry 2001;58: 229-30.

[12] Hemingway H, Marmot M. Evidence based cardiology - psychosocial factors in the aetiology and prognosis of coronary heart disease: systematic review of prospective cohort studies. BMJ 1999;318: $1460-7$.

[13] Musselman DL, Marzec U, Davidoff M, et al. Platelet activation and secretion in patients with major depression, thoracic aortic atherosclerosis, or renal dialysis treatment. Depress Anxiety 2002;15:91101.

[14] Kop WJ, Gottdiener JS, Tangen CM, et al. Inflammation and coagulation factors in persons $>65$ years of age with symptoms of depression but without evidence of myocardial ischemia. Am J Cardiol 2002;89:419-24.

[15] Kawachi I, Sparrow D, Vokonas PS, Weiss ST. Symptoms of anxiety and risk of coronary heart disease - the Normative Aging Study. Circulation 1994;90:2225-9.

[16] Kubzansky LD, Kawachi I, Weiss ST, Sparrow D. Anxiety and coronary heart disease: a synthesis of epidemiological, psychological, and experimental evidence. Ann Behav Med 1998;20:47-58.

[17] Sareen J, Cox BJ, Clara I, Asmundson GJG. The relationship between anxiety disorders and physical disorders in the US National Comorbidity Survey. Depress Anxiety 2005;21:193-202.

[18] Barger SD, Sydeman SJ. Does generalized anxiety disorder predict coronary heart disease risk factors independently of major depressive disorder? J Affect Disord 2005;88:87-91.

[19] National Center for Health Statistics. Evaluation of National Health Interview Survey diagnostic reporting. Vital Health Stat 2 1994;120: $1-116$.

[20] Kriegsman DMW, Penninx BWJH, Eijk JThMv, Boeke AJP, Deeg DJH. Self-reports and general practitioner information on the presence of chronic diseases in community dwelling elderly. A study on the accuracy of patients' self-reports and on determinants of inaccuracy. J Clin Epidemiol 1996;49:1407-17.

[21] Kehoe R, Wu SY, Leske MC, Chylack Jr LT. Comparing self-reported and physician-reported medical history. Am J Epidemiol 1994;139: $813-8$.

[22] Tretli S, Lund-Larsen PG, Foss OP. Reliability of questionnaire information on cardiovascular disease and diabetes: cardiovascular disease study in Finnmark County. J Epidemiol Community Health 1982;36:269-73.

[23] Kolk AM, Hanewald GJ, Schagen S, Gijsbers van Wijk CM. Predicting medically unexplained physical symptoms and health care utilization. A symptom-perception approach. J Psychosom Res 2002;52:35-44.

[24] Bergmann MM, Byers T, Freedman DS, Mokdad A. Validity of selfreported diagnoses leading to hospitalization: a comparison of selfreports with hospital records in a prospective study of American adults. Am J Epidemiol 1998;147:969-77.

[25] Kessler RC, Berglund P, Chiu WT, et al. The US National Comorbidity Survey Replication (NCS-R): design and field procedures. Int J Methods Psychiatr Res 2004;13:69-92.

[26] Kessler RC, Ustun TB. The World Mental Health (WMH) Survey Initiative Version of the World Health Organization (WHO) Composite International Diagnostic Interview (CIDI). Int J Methods Psychiatr Res 2004;13:93-121. 
[27] American Psychiatric Association. Diagnostic and statistical manual of mental disorders, fourth edition (DSM-IV). Washington DC: American Psychiatric Association; 1994.

[28] Penninx BWJH, Beekman ATF, Ormel J, et al. Psychological status among elderly people with chronic diseases: does type of disease play a part? J Psychosom Res 1996;40:521-34.

[29] Stansfeld SA, Fuhrer R, Shipley MJ, Marmot MG. Psychological distress as a risk factor for coronary heart disease in the Whitehall II Study. Int J Epidemiol 2002;31:248-55.

[30] Yates WR, Mitchell J, Rush AJ, et al. Clinical features of depressed outpatients with and without co-occurring general medical conditions in STAR*D. Gen Hosp Psychiatry 2004;26:421-9.

[31] Ormel J, Kempen GIJM, Penninx BWJH, Brilman EI, Beekman ATF, van Sonderen E. Chronic medical conditions and mental health in older people: disability and psychosocial resources mediate specific mental health effects. Psychol Med 1997;27:1065-77.

[32] Wolter KM. Introduction to variance estimation. New York, NY: Springer-Verlag; 1985.

[33] Research Triangle Institute. SUDAAN (Version 8.0.1). Research Triangle Park, NC: Research Triangle Institute; 2002.

[34] van Belle G, Fisher LD, Heagerty PJ, Lumley T. Biostatistics: a methodology for the health sciences. 2nd ed. Hoboken, New Jersey: Wiley; 2004.
[35] Bird SM, Cox D, Farewell VT, Goldstein H, Holt T, Smith PG. Performance indicators: good, bad, and ugly. J Royal Stat Soc A Series 2005;168:1-27.

[36] DiMatteo MR, Lepper HS, Croghan TW. Depression is a risk factor for noncompliance with medical treatment - meta-analysis of the effects of anxiety and depression on patient adherence. Arch Int Med 2000;160:2101-7.

[37] Klimes I, Mayou RA, Pearce MJ, Coles L, Fagg JR. Psychological treatment for atypical noncardiac chest pain - a controlled evaluation. Psychol Med 1990;20:605-11.

[38] Cannon III RO, Quyyumi AA, Mincemoyer R, et al. Imipramine in patients with chest pain despite normal coronary angiograms. N Engl J Med 1994;330:1411-7.

[39] Yusuf S, Reddy S, Ounpuu S, Anand S. Global burden of cardiovascular diseases: Part II. Variations in cardiovascular disease by specific ethnic groups and geographic regions and prevention strategies. Circulation 2001;104:2855-64.

[40] Havenaar JM, Poelijoe NW, Kasyanenko AP, van den Bout J, Koeter MW, Filipenko VV. Screening for psychiatric disorders in an area affected by the Chernobyl disaster: the reliability and validity of three psychiatric screening questionnaires in Belarus. Psychol Med 1996;26: 837-44. 